\title{
EMPYEMA THORACIS OF ODONTOGENIC ORIGIN: EXPERIENCE FROM A MAXILLOFACIAL UNIT
}

\author{
Benjamin Fomete', EzekielT. Adebayo², Muhammad B. Aminu³, Sunday 0. Ajike \\ 'Department of Maxillofacial Surgery, Ahmadu Bello University Teaching Hospital, Zaria, Nigeria \\ 2Department of Oral and Maxillofacial Surgery, University of Medical Sciences, Ondo, Nigeria \\ ${ }^{3}$ Division of Cardiothoracic Surgery, Department of Surgery, Ahmadu Bello University Teaching Hospital, Zaria, Nigeria
}

\begin{abstract}
INTRODUCTION: Cervicofacial infections of odontogenic origin continue to cause various complications despite improvements in clinical services, especially the use of potent antibiotics. Empyema thoracis is a life-threatening and extremely rare complication of odontogenic infections.

ОвјестіVEs: The aim of this report is to discuss the pattern of presentation of empyema thoracis as complication of odontogenic cervicofacial infections observed in a maxillofacial unit.

MATERIAL AND METHODS: A retrospective review of cases of descending cervicofacial infection with chest cavity involvement seen at the oral and maxillofacial clinic and co-managed with the cardiothoracic unit between January 2016 and January 2020

RESULTS: Five male patients presented with empyema thoracis from odontogenic infections, aged between 21 and 29 years (median, 27; mean age, 25.4 years). Coexisting systemic conditions were diabetes mellitus (1 patient), malnutrition (1 case), and mental illness (1 patient). In all cases, plain radiographs revealed pneumothorax, lung collapse, and pleural effusion. Treatment included systemic intravenous antibiotic therapy, control of diabetes, nutritional supplementation, and chest tube insertion. There was favorable outcome in four cases, while one patient died. Patients were discharged home after removal of chest tube.

Conclusions: Cervicofacial odontogenic infections are complicated by empyema thoracis, which could be fatal in various patients.
\end{abstract}

KEY WORDS: empyema thoracis, odontogenic infection, complication.

J Stoma 2020; 73, 3: 107-111

DOI: https://doi.org/10.5114/jos.2020.96758

\section{INTRODUCTION}

Cervicofacial odontogenic infections are serious conditions that can spread upwards to the brain or downwards into the pleural and mediastinal cavities. Downwards spread through the cervical spaces into the pleural cavity with pus collection known as empyema thoracis is exceedingly rare [1], even though, em- pyema was firstly diagnosed by Hippocrates over 2,000 years ago.

Despite the availability of antibiotics, cervicofacial infections of odontogenic origin can cause various local and systemic complications from hematogenous, lymphatic, or direct spread due to ignorance of patients and other factors. Even though such complications are self-limiting, they sometimes lead to significant mor-

\section{JOURNAL OF} STOMATOLOGY CZASOPISMO STOMATOLOGICZNE

AdDRESS FOR CORRESPONDENCE: Benjamin Fomete, Maxillofacial Surgery Department, Ahmadu Bello University Teaching Hospital, Nigeria, e-mail: benfometey@hotmail.com

RECEIVED: 13.05.2020 • ACCEPTED: 09.06.2020 • PUBLISHED: 30.06.2020 
bidity or even death [2]. Descending neck infections are rarely complicated by empyema thoracis. The outcome of this complication depends on early diagnosis and aggressive treatment [3]. However, there are few case series on the pattern of presentation and the treatment of complication from a resource-constrained environment.

\section{OBJECTIVES}

The aim of this report was to discuss the pattern of presentation of empyema thoracis as a complication of odontogenic cervicofacial infections in a resource-constrained environment.

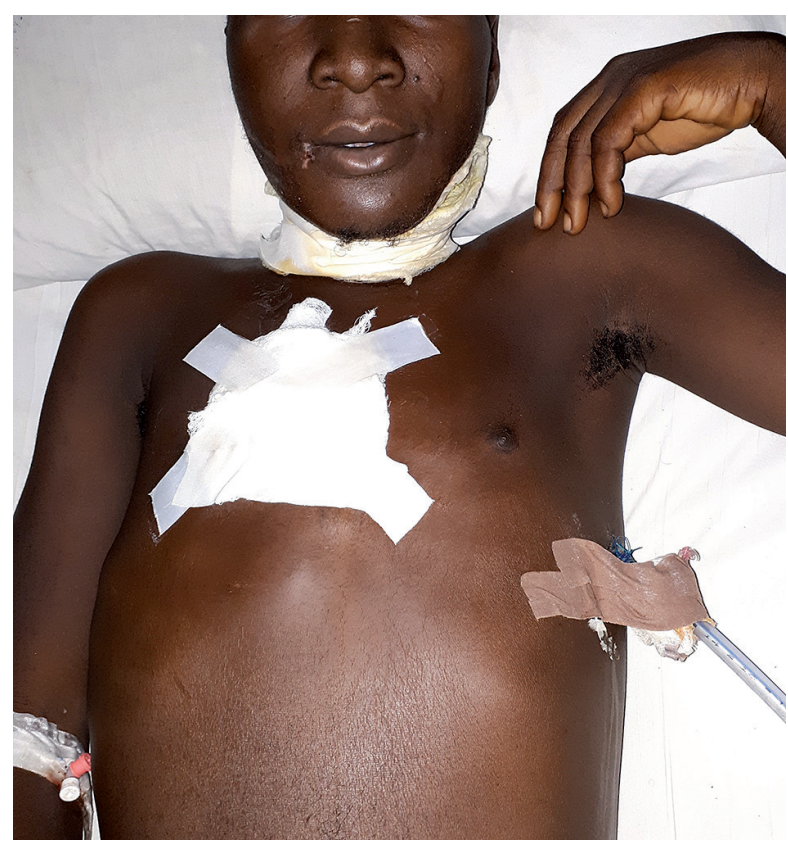

FIGURE 1. Male diabetic patient with a chest tube on the left

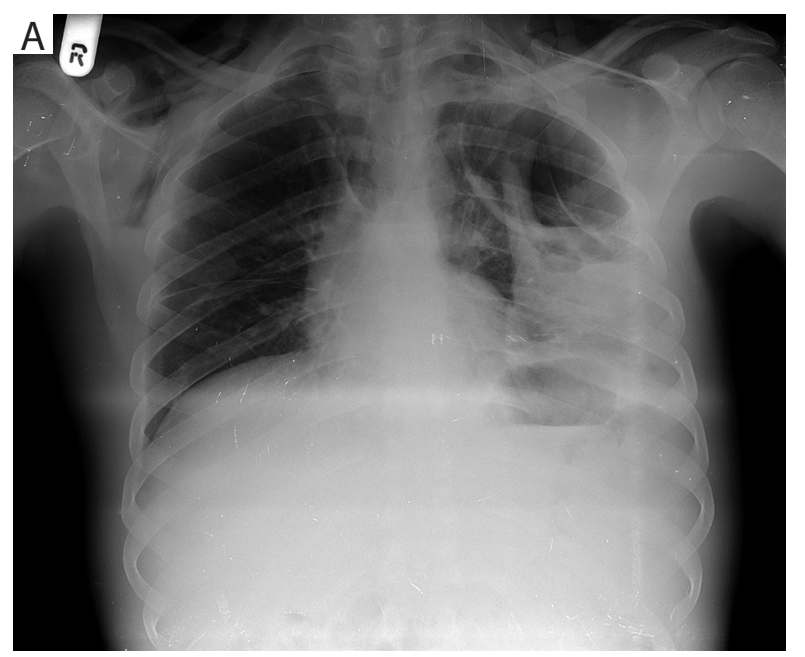

\section{MATERIAL AND METHODS}

Retrospective review of cases of descending cervicofacial infection with chest cavity involvement observed at the oral and maxillofacial clinic and comanaged with the cardiothoracic unit between January 2016 and January 2020 was undertaken. Data collected from medical folders included age, sex, diagnosis, investigations, presentation and treatment of dental infections, and management of chest infections, duration of hospital stay, quantity of fluid drained, and outcome of treatments.

\section{RESULTS}

Five cases of empyema thoracis caused by odontogenic infections were studied. All were males, age range 21 to 29 years (median, 27 years; mean age, 25.4 years). Coexisting, pre-existing systemic illnesses were diabetes mellitus (1 case), malnutrition with nutritional anemia (1 patient), and a psychiatric illness ( 1 case) (Figure 1$)$. Dental caries was the precipitating cause of the cervicofacial infection in all studied cases. Dental intraoral periapical radiographs showed periapical abscesses related to the cervicofacial infection, but some cases of empyema thoracis occurred on the opposite side to the offending tooth. Chest side affected were the right side ( 3 cases) and the left side ( 2 cases). Chest findings included increased respiratory rate, tracheal deviation to the opposite side, reduced chest wall expansion, reduced tactile fremitus, reduced percussion note, diminished breath sounds, and vocal resonance on the infraclavicular and infra-axillary regions. Chest radiograph (posteroanterior view) was carried out, which showed pneumothorax, lung collapse, and pleural effusion on the affected side (Figure 2). Our patients had no finance for other radiological tests like computed tomography (CT), which was available in the hospital.

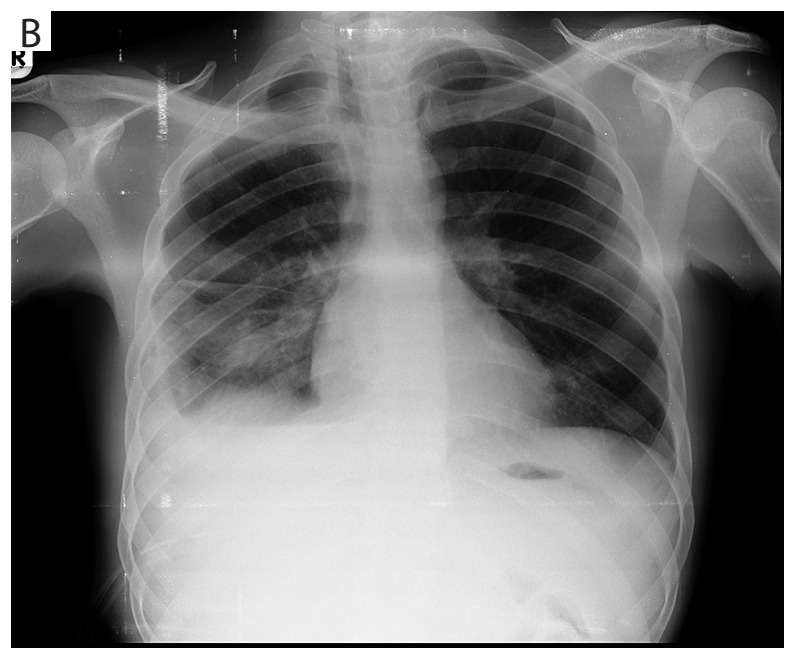

FIGURE 2. Chest radiographs of a patient before $(\mathbf{A})$ and at the end of the treatment (B) 
Multidisciplinary management of patients included an endocrinologist for management of diabetes mellitus, a nutritionist, who provided high protein fluid diet (kwashi pap) for malnutrition and intravenous antibiotics (penicillin, metronidazole, and gentamycin), while a psychiatrist was requested to manage the patient with psychiatric issues. Extraction of the offending tooth/ teeth was done as soon as the patient's condition improved. Cardiothoracic surgeons carried out thoracocentesis, followed by thoracotomy with chest tube insertion, and the pus was submitted for pleural fluid analysis. The microbiology and culture yielded no growth; anaerobic culture was not performed. Therefore, patients were continued on previously prescribed empirical antibiotics. The median initial volume of pus collected with the chest tube was $1,000 \mathrm{ml}$ (range, 750-1,700 ml); a week later, median aspirate was $500 \mathrm{ml}$ (range, 300-1,000 ml). Chest tube was in situ between 21 and 56 days (median, 35 days). Overall, four patients had satisfactory outcomes and were discharged home after the removal of chest tube, while one patient (Figure 3) died three days after the insertion of chest tube.

\section{DISCUSSION}

This study presented five cases of empyema thoracis as a complication of odontogenic infections observed over four years, which indicates rarity of the condition. The present series of five cases is the highest ever published to our knowledge. The total number of reported cases of empyema thoracis from our center is now $7[3,5]$.

Previous reports indicate that thoracic complications of odontogenic infections have no age preference. Nigerian cases [3-5] have been young adults; all our five patients were between 21-29 years old. Most cervicofacial infections that descend into the neck and chest are caused by tooth infections, especially dental and periodontal abscesses [2, 6]. All our patients had dentoalveolar abscess from untreated dental caries, resulting in fascial space infections that tracked into the pleural cavities. These reinforce the need for prompt recognition and the treatment of dental conditions to avoid spreading fascial space infections. Improved dental education and awareness combined with the access to prompt care can reduce the incidence of complication from dental conditions.

In economically developed countries, the prevalence of human immunodeficiency virus (HIV)/acquired immunodeficiency syndrome (AIDS), wide use of immune suppressants, organ transplantation, and ageing of population have been identified as predisposing factors for the development of empyema [7]. In our environment, conditions that depress the immune system, such as uncontrolled diabetes mellitus and malnutrition, were observed in some of our patients. A case reported by Ibikunle et al. [5] had no identified immune deficit.

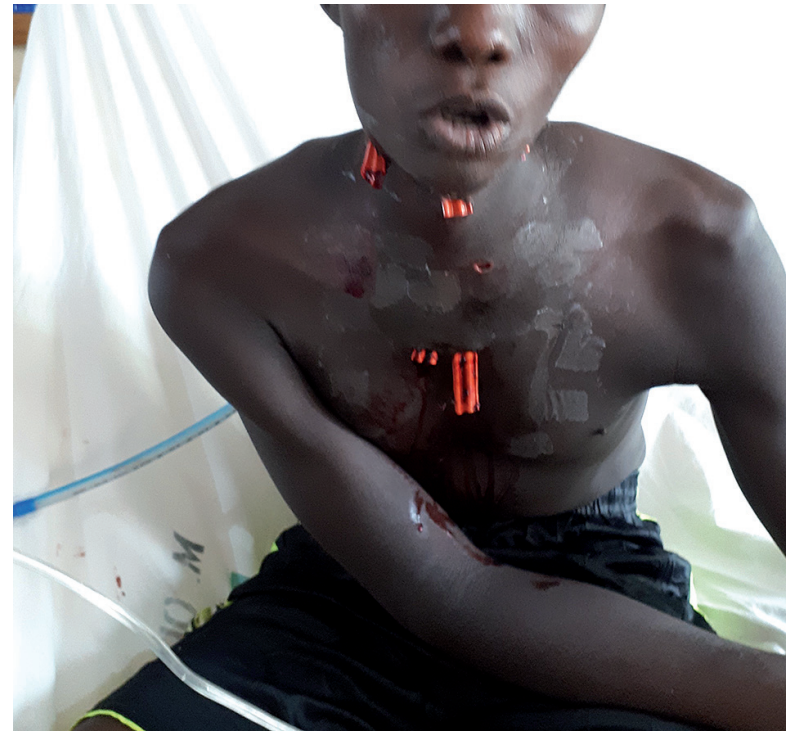

FIGURE 3. Male patient with psychiatric issues

Aerobic pneumonic infections usually present with acute febrile illness, localized pleuritic chest pain, sputum production, and leukocytosis. Moreover, these infections often have a more insidious course, with less severe fever but general systemic symptoms, such as poor appetite and weight loss. Anaerobic infections are more common among those with poor dental hygiene [8]. Our patients' health conditions developed from the cervicofacial ailment to empyema within 5 to 14 days, which indicates an insidious onset.

Radiological investigations are important in managing both empyema thoracis and cervicofacial infection. These investigations range from plain radiographs, ultrasound scans, CT, and magnetic resonance imaging (MRI) $[4,5]$. Radiologic findings of obliteration of the costophrenic angle and tracheal deviation in the presence of suspicious clinical findings on chest examination would prompt the need for other tests to identify the location of pus to guide patient's management [7]. In our environment, the affordability of hospital care services is a serious challenge, as most patients need to pay out of pocket for hospital services.

Treatment of these patients is multidisciplinary and includes an improvement in blood glucose control and correction of malnutrition. Empirical antibiotics are also administered based on known cervicofacial infection antimicrobial sensitivity patterns. The antibiotics utilized for our patients are similar to those earlier reported by Fomete et al. [4] and Ibikunle et al. [5]. Hippocrates believed that pus in the pleura must either be let out or would kill the patient [7]. Our patients had an evacuation of pus through thoracocentesis and thoracotomy. The median initial volume of pus recorded in our patients was 1,000 ml. Lower volumes (400 $\mathrm{ml}$ and $650 \mathrm{ml}$ ) were reported by Agbara et al. [3] and Glen and Morison [9], but a higher volume $(1,800 \mathrm{ml})$ has also been 
TABLE 1. Clinical details of patients with empyema thoracis

\begin{tabular}{|c|c|}
\hline Characteristic & Total (\%) \\
\hline No of patients & $5(100 \%)$ \\
\hline \multicolumn{2}{|l|}{ Demographics } \\
\hline Age (years), mean (range) & $25.4(21-29)$ \\
\hline \multicolumn{2}{|l|}{ Sex, M/F (ratio) } \\
\hline \multicolumn{2}{|l|}{ Clinical features } \\
\hline 1. 28 yrs old with descending cervicofacial infection down to the chest related to lower Lt 7 ; breathlessness, pain, fever & Lt thoracotomy \\
\hline $\begin{array}{l}\text { 2. } 27 \text { yrs with Rt hemifacial swelling crossing to the Lt and descending to the anterior chest wall. Infection related to } \\
\text { lower Rt } 8 \text { and Lt } 7\end{array}$ & Rt thoracotomy \\
\hline $\begin{array}{l}\text { 3. } 22 \text { yrs with bilateral cervicofacial infection descending to the anterior chest wall up to the level of } 4^{\text {th }} \text { rib, associated } \\
\text { breathlessness }\end{array}$ & Lt thoracotomy \\
\hline 4. $29 \mathrm{yrs}$, recurrent toothache, swelling extending to the sternoclavicular junction, fever, pain, carious lower Rt 7 & Rt thoracotomy \\
\hline $\begin{array}{l}\text { 5. } 21 \text { yrs, recurrent Rt submandibular swelling now extending to the Lt and down to the } 5 \text { th rib anterior chest wall; } \\
\text { related to lower Lt 6, breathlessness, restlessness, irrational behavior }\end{array}$ & Rt thoracotomy \\
\hline \multicolumn{2}{|l|}{ Side } \\
\hline Rt/Lt (ratio) & $3: 2$ \\
\hline Swelling (mean in days) & 9.5 (5-14 days) \\
\hline Toothache (mean in days) & $15.4(7-28)$ \\
\hline Tooth involved & Lower molars \\
\hline Initial drainage (mean volume) & $1,190 \mathrm{ml}(750-1,700 \mathrm{ml})$ \\
\hline A week late (mean volume) & $450 \mathrm{ml}(300-1,000 \mathrm{ml})$ \\
\hline Length of stay (mean in days) & 35 (21-56 days) \\
\hline Comorbidities: diabetes mellitus (1), malnutrition and anemia (1), psychiatric illness (1) & 2 \\
\hline
\end{tabular}

reported [1]. The volume of pus aspirated is an indication of severity of infection, and the fact that two of our patients were malnourished could explain the higher median volume of aspirate in our study.

In the present study, there were no predilection for the chest side affected, despite the anatomical fact that the right bronchus has less angulation to the lung than the left bronchus. The absence of side predilection could also be an indicator of rarity of the condition. Earlier Nigerian cases had chest tube insertion on the right side [5] and on the left side [3]. Three of the five patients (60\%) in our series had chest tube insertion on the right side. Glen and Morison [9] reported the left side. It was also observed that empyema can occur on the side contralateral to the dental infection, possibly through the release of infective emboli. One patient in our series had bilateral dental foci of the infection with right-sided empyema, while another had contralateral chest involvement.

Conventional laboratory methods have a low yield ( $60 \%)$ of bacterial culture from pleural aspirates in empyema [10]. In our study, all aspirates from the five patients yielded no growth. Likely reasons for this finding could be an effective antibiotic treatment prior to sample collection, or that the ongoing inflammatory process did not need the continued presence of bacteria for its sus- tenance. Other possibilities are lack of sensitivity of conventional culture techniques and presence of pleural biofilm [10].

All thoracic drainages were performed using underwater sealed chest tube. This was also used by Ibikunle et al. [5]. Other methods include the use of indwelling catheters and video-assisted thoracotomy [7]. The disadvantage with underwater sealed chest tube is that about $20 \%$ of such patients still require open thoracotomy.

The mean duration of hospital stay was 21 days, which is comparable with previous reports $[1,5]$. The longest staying patient (56 days) was the one diagnosed with diabetes mellitus. Two nutritional anemic patients had an improvement in hematologic parameters before discharge (one was transfused, and both were given kwashi pap and hematinic). Overall, most patients recovered satisfactorily and were discharged, while there was one fatality.

\section{CONCLUSIONS}

Cervicofacial infections of odontogenic origin can result in rare but profoundly serious complications, such as empyema thoracis, especially in immunosuppressed patients. The need for multidisciplinary management to 
reduce morbidity and mortality from these complications is re-emphasized. This case series show the necessity for prevention of severe cervicofacial odontogenic infections, prompt and effective recognition, and treatment of complications like empyema, to reduce morbidity and prevent mortality.

\section{CONFLICT OF INTEREST}

The authors declare no potential conflicts of interest with respect to the research, authorship, and/or publication of this article.

\section{References}

1. Juretic M, Belusic-Gobic M, Kukuljan M, Cerovic R, Golubovic V, Gobic D. Mediastinitis and bilateral pleural empyema caused by an odontogenic infection. Radiol Oncol 2007; 41: 57-62.

2. Sawalha W, Ahmad M. Bilateral pleural empyema following pericoronitis. Ann Saudi Med 2001; 21: 3-4.

3. Agbara R, Obiadazie AC, Ediagbini S, Ikekhuamen E. A fatal case of empyema thoracis: the price for underestimating odontogenic infections. Oral Maxillofac Surg Cases 2016; 2: 22-26.

4. Fomete B, Agbara R, Osunde OD, Ononiwu CN. Cervicofacial infection in a Nigerian tertiary health institution: a retrospective analysis of 77 cases. J Korean Assoc Oral Maxillofac Surg 2015; 41: 293-298

5. Ibikunle AA, Taiwo AO, Braimah RO, Abubakar U. Empyema thoracis: a life-threatening complication of odontogenic infection. J Med Soc 2018; 32: 150-153.

6. Marioni G, Rinaldi R, Staffieri C, et al. Deep neck infections with dental origin: analysis of 85 consecutive cases (2000-2006). Acta Otolaryngol 2008; 128: 201-206.

7. Brims FJ, Lansley SM, Waterer GW, Lee YC. Empyema thoracis: new insights into an old disease. Eur Respir Rev 2010; 19: 220-228.

8. Bartlett JG, Gorbach SL, Thadepalli H, et al. Bacteriology of empyema. Lancet 1974; 1: 338-340.

9. Glen P, Morrison J. Diffuse descending necrotizing mediastinitis and pleural empyema secondary to acute odontogenic infection resulting in severe dysphagia. BMJ Case Rep 2016. doi:10.1136/ bcr-2015-212145.

10. Ahmed RA, Marrie TJ, Huang JQ. Thoracic empyema in patients with community-acquired pneumonia. Am J Med 2006; 119: 877 883. 